\title{
Single Center Experience in the Surgical Management of Primary Hyperparathyroidism
}

\author{
Berat Demir ${ }^{1}$ (i) $\cdot$ Adem Binnetoglu² [i] $\cdot$ Akın Sahin ${ }^{1}$ (i) $\cdot$ Dilek Gogas Yavuz ${ }^{3}$ (i) \\ ${ }^{I}$ Department of Otorhinolaryngology-Head and Neck Surgery, Pendik Training and Research Hospital, Marmara University Medical Faculty, \\ Istanbul, Turkey; ' Department of Otorhinolaryngology-Head and Neck Surgery, St. Elizabeth's Medical Center, Brighton, MA, USA; \\ ${ }^{3}$ Section of Endocrinology and Metabolism, Pendik Training and Research Hospital, Marmara University Medical Faculty, Istanbul, Turkey
}

Objectives. As calcium included as a part of routine laboratory screening early diagnosis of primary hyperparathyroidism (PHPT) has been increased. Surgical resection of parathyroid adenoma or hyperplasia still is the mainstay of the treatment for most PHPT patients. The aim of this study was to evaluate of the surgical outcomes of patients with PHPT that referred to our ENT department of our University Hospital for the last 6 years.

Methods. One hundred thirty-seven patients with PHPT who underwent parathyroid surgery in our clinic between October 2011 and January 2018 included in this retrospective study. Data on demographics, clinical findings, past medical history, preoperative laboratory values in 3 months, preoperative localizing imaging studies including ultrasonography (USG) and 99mTc-sestamibi (methoxyisobutyl isonitrile, [MIBI]) scan, operative findings, postoperative laboratory values, and pathology reports were recorded. MIBI scan and USG are used as the first-line modalities in our center. Single-photon emission computed tomography was used for challenging situations of re-exploration and ectopic parathyroid pathology. Four-dimensional computed tomography scanning is was preferred as the last imaging modality. Focused unilateral neck exploration (FUNE) was performed with intraoperative frozen section analysis as a routine procedure. Bilateral neck exploration (BNE) was used only in re-exploration, ectopic parathyroid, and with high suspicion of multigland disease.

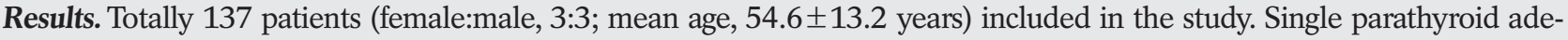
noma was found in 108 patients $(78.8 \%)$. Most common adenoma localization was left inferior parathyroid gland (46.7\%). FUNE was performed in $89.8 \%$ of the patients and BNE for $10.2 \%$ of the patients. Postoperative normocalcemia was reached in 132 patients and permanent hypocalcemia was observed in two patients. Persistence hypercalcemia observed in three patients. Postoperative pathology reports revealed three patients have parathyroid carcinoma.

Conclusion. Preoperative imaging modalities is very important in parathyroidectomy surgery. Routine use of preoperative imaging modalities reduced the risk of complications in our clinic.

Keywords. Primary Hyperparathyroidism; Parathyroidectomy; Bilateral Neck Exploration; Four-Dimensional Computed Tomography Scanning

\section{INTRODUCTION}

- Received August 21, 2019

Revised September 30, 2019

Accepted November 13, 2019

- Corresponding author: Berat Demir

Department of Otorhinolaryngology-Head and Neck Surgery, Marmara

University Medical Faculty, Mimar Sinan Caddesi No. 41, Fevzi Cakmak

Mahallesi, Ust Kaynarca-Pendik, 34899 Istanbul, Turkey

Tel: +90-216-625-45-45, Fax: +90-216-625-46-39

E-mail: drberatdemir80@hotmail.com
Primary hyperparathyroidism (PHPT) is the most common cause of the hypercalcemia. As blood calcium measurements became a part of routine laboratory evaluation in clinics, diagnosis of asymptomatic hyperparathyroidism has been increased. Recent literature reported that $75 \%-80 \%$ of PHPT is caused by parathyroid adenoma followed, by primary parathyroid hyperplasia $(15 \%-20 \%)$ [1]. Surgery is the treatment of choice for most

Copyright (C) 2020 by Korean Society of Otorhinolaryngology-Head and Neck Surgery.

This is an open-access article distributed under the terms of the Creative Commons Attribution Non-Commercial License (https://creativecommons.org/licenses/by-nc/4.0)

which permits unrestricted non-commercial use, distribution, and reproduction in any medium, provided the original work is properly cited. 
PHPT patients [2]. Parathyroid surgery is a reliable in experienced hands and provides cure for more than $95 \%$ of patients [3]. The classic bilateral neck exploration (BNE) technique that four glands were found and resection of those that were enlarged, was used for many years. There was a transition from BNE to minimal invasive techniques with the advancement of imaging modalities. Nowadays, minimally invasive parathyroidectomy is more preferred method among many surgical methods, especially in patients whose pathology is localised by preoperative imaging modalities. Although the long-term surgical success rates of both techniques is the same, relatively less dissection (minimizing the risk of recurrent laryngeal nerve damage or devascularization of normal parathyroid glands), making it faster with smaller incisions, reducing hospital costing leads surgeons to this technique [4-6]. BNE is only used in re-exploration, ectopic parathyroid, and in those with high suspicion of multigland disease. We have increased amount of PHPT patients referred to our clinic for operation. As a routine procedure, focused unilateral neck exploration (FUNE) was performed with intraoperative frozen section analysis aiming to decrease postoperative complications. BNE was only used in re-exploration, ectopic parathyroid, and with high suspicion of multigland disease. In this retrospective study we aimed to evaluate clinical features, clinical outcomes in our group of PHPT patients referred to our ENT clinic and underwent for PHTP surgery between 2011 and 2018.

\section{MATERIALS AND METHODS}

The Ethical Committee of Marmara University's Faculty of Medicine approved the study and informed consent was obtained from the patients. All procedures performed in the study were in accordance with the ethical standards of this research committee. Informed consent in written was received. All 137 Patients diagnosed PHPT and operated to our ENT department between 2011 and 2018 were included in the study. All patients with PHPT were referred to the ENT department after evaluation in Marmara University's Endocrinology and Metabolism Department.

\section{H I G H L I G G H T S}

- Primary hyperparathyroidism is the most common cause of the hypercalcemia.

- Minimally invasive parathyroidectomy is more preferred method among many surgical methods.

- Bilateral neck exploration was only used in re-exploration, ectopic parathyroid, and with high suspicion of multigland disease.

- Four-dimensional computed tomography scanning is was preferred as the last imaging modality that pathology could not be detected with ultrasonography and $99 \mathrm{mTc}$-sestamibi.
Data collected form patients files retrospectively. The diagnosis of PHPT is based on laboratory finding including serum total calcium, intact parathyroid hormone (iPTH), phosphate, urine calcium excretion. Surgery was recommended to all asymptomatic patients who fulfilled the criteria according to National Institutes of Health 2009 consensus guidelines indications [2] such as serum calcium at more than $1 \mathrm{mg} / \mathrm{dL}$ above the upper limits of normal, T-score $<-2.5$ at any site for bone mineral density and/or previous fragility fracture, previous fragility fracture, estimated glomerular filtration rate $<60 \mathrm{~mL} / \mathrm{min}$, age $<50$ years. In addition, parathyroidectomy was performed in symptomatic patients, although the age $>50$ years.

Pre- and posttreatment clinical, laboratory and radiologic data as well as surgery notes were recorded. Serum calcium, phosphate, iPTH, 25-hydroxyvitamin D, alkaline phosphatase, creatinine levels were recorded at preoperative and postoperative 1 day, 6 and 12 months. After biochemical confirmation preoperative radiologic evaluations with ultrasonography (USG), methoxyisobutyl isonitrile (MIBI) were recorded. Single-photon emission computed tomography (SPECT) was used for challenging situations of re-exploration and ectopic parathyroid pathology. Fourdimensional computed tomography (4D-CT) scanning was preferred as the last imaging modality that pathology could not be detected with USG and MIBI. Clinical parameters presence of diabetes hypertension osteoporosis, renal stones collected as well as postoperative pathology reports.

\section{Surgical technique}

The patients underwent parathyroid surgery with the aim of complete resection of the parathyroid pathology. Focused exploration of the neck in patients with PHPT was performed under general anesthesia using endotracheal intubation. All patients had modified Conley incisions. BNE was used in re-exploration, ectopic parathyroid, and in those with high suspicion of multigland disease. Modified Kocher incisions were used in this procedure, which are low transverse cervical incisions approximately $4 \mathrm{~cm}$ in length, designed two fingerbreadths above the suprasternal notch, usually over a natural skin crease, and is carried down through the platysma.

Patients with solitary adenomas, after the enlarged, abnormal gland was identified, it was resected with an intact capsule. In the cases of four gland hyperplasia, after exploration of all hyperplastic glands, the three largest glands were removed and histologically confirmed. Subtotal excision of the remaining gland follows, leaving at least one-third to one-half of the gland as a viable vascularized remnant.

For re-exploration and ectopic parathyroid patients, a gamma radiation detection device was used with the assistance of a nuclear radiologist who actively participated during the surgery. All patients who underwent revision surgery had samples sent for intraoperative frozen section analysis for confirmation of the parathyroid tissue. Intraoperative parathyroid hormone (IoPTH) 
Table 1. Preoperative and postoperative laboratory results

\begin{tabular}{lcccc}
\hline Variable & Preoperative & Postoperative 1 day & Postoperative 6 months & Postoperative 12 months \\
\hline Calcium $(\mathrm{mg} / \mathrm{dL})$ & $11.74 \pm 1.08$ & $9.06 \pm 0.9$ & $8.86 \pm 1.22$ & $8.62 \pm 1.22$ \\
Phosphate $(\mathrm{mg} / \mathrm{dL})$ & $2.57 \pm 0.69$ & $3.17 \pm 0.52$ & $3.13 \pm 0.27$ & $3.15 \pm 1.07$ \\
PTH $(\mathrm{pg} / \mathrm{mL})$ & $332.74 \pm 368.62$ & $58.59 \pm 11.1$ & $59.80 \pm 34.6$ & $52.13 \pm 197.29$ \\
\hline
\end{tabular}

Values are presented as mean \pm standard deviation.

PTH, parathyroid hormone.

assay was not used as an intraoperative adjunct.

All patients were considered for discharge 24-48 hours after surgery. PTH and calcium levels were checked at day 1, 6 and 12 months postoperatively. All patients were seen in the outpatient clinic at 1 week, 6 and 12 months after surgery, and were assessed for symptoms, complications, and final histologic diagnosis. Serum PTH and calcium levels were measured at each visit. Pathologic diagnosis was recorded for each patient. Persistent hypercalcaemia was accepted as recurrent presence of high calcium within 6 months of operation.

\section{Statistical analysis}

Statistical analyses were carried out using the SPSS ver. 15.0 (SPSS Inc., Chicago, IL, USA). For the parametric distribution of values, Student $t$-test was used to compare the mean values of 2 or more groups. The results were given mean \pm standard derivation and they were considered significant at a $P$-value of less than 0.05 .

\section{RESULTS}

There were 137 patients (female:male, 3:3) in our study. The mean age was $54.6 \pm 13.2$ years. FUNE was the surgical method that we preferred in 123 patients $(89.8 \%)$. BNE was performed in 14 patients $(10.2 \%)$ including five patients with revision surgery, three patients with ectopic localization and six patients with high suspicion of multigland disease. Most patients with PHPT were found to be asymptomatic at presentation $(n=97$, $66.3 \%$ ). Thirteen patients had nephrolithiasis, 10 had hypertension, seven had bone pain, six had type 2 diabetes mellitus, one had osteoporosis, one had anxiety.

One hundred and eight patients had benign pathology of adenoma, 16 had hyperplasia and three had parathyroid carcinoma, three patient had normal parathyroid tissue, two had atypical adenoma, one had parathyroid hyperplasia, and one had no parathyroid tissue. All patients received preoperative both USG and scintigraphy examinations. All patients except five patients have normocalcemia postoperatively. In these five patients, the pathology was not determined by both USG and computed tomography examinations. Revision surgery was performed on these five patients using BNE. In 10 of 137 patients, pathology could not be localized with preoperative imaging techniques (USG and scintigraphy). Five of these ten patients underwent re-
Table 2. Anatomical localizations of parathyroid pathologies

\begin{tabular}{lcc}
\hline Anatomical localization & $\begin{array}{c}\text { Primary } \\
\text { surgery }\end{array}$ & $\begin{array}{c}\text { Revision } \\
\text { surgery }\end{array}$ \\
\hline Left inferior adenoma & $64(46.7)$ & - \\
Right inferior adenom & $34(24.8)$ & $1(20)$ \\
Right superior adenoma & $13(9.5)$ & $1(20)$ \\
No uptake & $10(7.3)$ & - \\
Four gland hyperplasia & $4(2.9)$ & - \\
Isthmus & $3(2.2)$ & $1(20)$ \\
Right lobe medium & $2(1.5)$ & - \\
Two gland hyperplasia & $2(1.5)$ & - \\
Mediastinum adenom & $1(0.7)$ & $1(20)$ \\
Intratorasic & $1(0.7)$ & - \\
Left inferior adenoma+left superior adenoma & $1(0.7)$ & - \\
Intratiroidal & - & $1(20)$ \\
\hline
\end{tabular}

Values are presented as number (\%).

vision surgery.

The mean preoperative serum calcium, phosphate, and PTH levels were $11.74 \pm 1.08 \mathrm{mg} / \mathrm{dL}, 2.57 \pm 0.69 \mathrm{mg} / \mathrm{dL}$, and $332.74 \pm$ $368.62 \mathrm{pg} / \mathrm{mL}$, respectively (Table 1). In patients with PHPT, the left inferior parathyroid gland was the most common site of adenomas (64 patients, $46.7 \%$ ). The right inferior parathyroid gland was the site in 34 patients $(24.8 \%$ ) (Table 2$)$.

The remainder of the adenomas were distributed as follows: the right superior in 13 patients $(9.5 \%)$, the isthmus in three $(2.2 \%)$, no uptake in $10(7.3 \%)$, the right lobe media in two $(1.5 \%)$, four-gland hyperplasia in four $(2.9 \%)$, two-gland hyperplasia in two $(1.5 \%)$, the mediastinum in one $(0.7 \%)$, both the left inferior and superior in one $(1 \%)$, and intrathoracic in one $(1 \%)$.

All patients received a preoperative both sestamibi scan and USG of the neck. While no pathology was observed in 17 patients by USG, scintigraphy was detected pathology in all of these patients. However, scintigraphy showed no uptake in ten patients. In addition, SPECT showed no uptake in seven of ten patients. Four-dimensional computed tomography scanning was performed in seven patients who could not be detected in either USG, MIBI, and SPECT. Four-dimensional computed tomography helped to find the adenoma in all of these seven patients.

The median follow-up time was 12 months. Postoperative serum calcium, phosphate, and PTH values were measured at postoperative day 1,6 , and 12 months after the surgeries for both primary and revision surgeries (Tables 1 and 3). Postoperative hypocalcemia was permanent in two patients. There were no 
Table 3. Postoperative serum calcium, phosphor and PTH values measured at 1 day, 6 and 12 months after revision surgeries

\begin{tabular}{lccc}
\hline $\begin{array}{l}\text { Postoperative revision } \\
\text { surgery }\end{array}$ & $\begin{array}{c}\text { Postopertive } \\
1 \text { day }\end{array}$ & $\begin{array}{c}\text { Postopertive } \\
6 \text { months }\end{array}$ & $\begin{array}{c}\text { Postopertive } \\
12 \text { months }\end{array}$ \\
\hline Calcium $(\mathrm{mg} / \mathrm{dL})$ & $8.83 \pm 1.1$ & $8.92 \pm 1.22$ & $8.71 \pm 1.19$ \\
Phosphate $(\mathrm{mg} / \mathrm{dL})$ & $3.28 \pm 0.34$ & $3.32 \pm 0.32$ & $3.32 \pm 0.23$ \\
PTH $(\mathrm{pg} / \mathrm{mL})$ & $88.67 \pm 14.56$ & $98.51 \pm 0.31$ & $98.67 \pm 197.2$ \\
\hline
\end{tabular}

Values are presented as mean \pm standard deviation.

PTH, parathyroid hormone.

a) $n=5$.

complications in 116 patients with PHPT. Transient hypocalcaemia was seen in 13 patients. Hungry bone syndrome occurred in five patients. Two patients had temporary unilateral and one patient had permanent unilateral cord paralysis. Bilateral cord paralysis was not observed in any patient. There were no wound infections or hematoma (Table 4). In patients with PHPT, 88 patients had no another thyroid pathology. Twenty-three had multinodular goitre, 19 patients had a single nodule, seven had thyroiditis. One patient's pathology resulted as intrathyroidal parathyroid adenoma but total thyroidectomy with parathyroidectomy was performed in that patient because of multinodular goitre. Total thyroidectomy was performed in one patient for his multinodular goitre, but his pathology resulted as thyroid papillary carcinoma.

\section{DISCUSSION}

We report our experience and outcomes of patients who underwent surgery for PHPT. Unlike the literature, nephrolithiasis was seen at a very low rate of $9.4 \%$ in in our group of patients with PHPT. Coffey et al. [7] attributed this to the increased rate of hypercalcaemia in routine blood tests and they reported that the rate of nephrolithiasis, which was $65 \%$ earlier, decreased to $29 \%$.

The optimal surgical approach in the treatment of patients with PHPT has become controversial in recent years due to focused parathyroid exploration. However, although BNE is the gold standard operation for sporadic PHT [8], FUNE has become the preferred approach with improvement in preoperative imaging. Hence, discussions about this issue have increased. Good cosmetic results, short operative time, short hospital stay, less pain and fewer postoperative complications make FUNE more attractive. We prefer FUNE in localized patients with preoperative methods. Even with this technique, we performed parathyroidectomy and thyroidectomy. We think that although preoperative imaging modalities indicates a single adenoma, surgeons should always be prepared to convert from a minimally invasive procedure into BNE if intraoperative findings show multigland disease.

Patients with negative preoperative imaging studies or a suspicion of multigland disease are certain indications for BNE. The use of IoPTH could avoid the need for BNE in many patients.
Table 4. Complications of parathyroidectomy

\begin{tabular}{lc}
\hline Variable & Value $(n=137)$ \\
\hline No complication & $116(84.7)$ \\
Transient hypocalcemia & $13(9.5)$ \\
Hypocalcemia and hungry bone syndrome & $5(3.6)$ \\
Temporary unilateral vocal cord paralysis & $2(1.4)$ \\
Permanent unilateral vocal cord paralysis & $1(0.7)$ \\
\hline
\end{tabular}

Values are presented as number (\%).

However, the experience of some groups with IoPTH in the presence of two or more enlarged glands raises the question of the significance of each gland in the hypersecretion of IoPTH [9]. Surgeons who routinely perform BNE with intraoperative parathormone monitoring have shown that although IoPTH levels can fall immediately after excision of the imaged-targeted gland, anatomically enlarged glands may remain in situ [9]. Until parathyroid enlargement and overproduction are better understood, surgeons must use their experience, judgment, and skills in determining the best course of action in each individual patient. Gasparri [9] stated that at least a decade was needed to compare BNE with focused surgeries using intraoperative PTH. The reason for this is that some of the glands may be in sleep during the operation and may be responsible for late recurrence. Norman et al. [10] gave up performing unilateral parathyroidectomy when they experienced a 1 -year failure rate of $3 \%-5 \%$ and a 10 -year recurrence rate of $4 \%-6 \%$ with this technique [10]. Besides, intraoperative PTH assays are extremely expensive. This is a major problem for developing countries such as Turkey. Horanyi et al. [11] reported that minimally invasive procedures might miss other overactive glands that are suppressed in the presence of a dominant gland. Therefore, they recommended other intraoperative methods such as histologic evaluation of frozen sections. The confirmation of the diagnosis was made using frozen-section examinations instead of IoPTH in our patients. The surgical procedure changes according to whether the pathology can be determined by preoperative imaging methods.

A universal algorithm for imaging parathyroid glands is still not available. The choice of imaging approach is largely dependent on the surgeon's preference and expertise in radiology. Nuclear medicine scintigraphy and USG are the most commonly used first-line modalities in medical practice. Dual-phase scintigraphy using a single radiopharmaceutical $(99 \mathrm{mTc}$ MIBI) or dualisotope subtraction scintigraphy using $99 \mathrm{mTc}$ MIBI and $99 \mathrm{mTc}-$ pertechnetate are scintigraphic techniques for the evaluation of PHPT.Tomographic imaging with SPECT or SPECT/CT increases the sensitivity of this modality. The sensitivity of USG and MIBI combined is higher than either technique alone. Although no imaging modality is superior to each other in the determination of parathyroid lesions, the popularity of 4D-CT scanning is increasing [12]. The source of PTH excess remains unclear in a 
small but important minority of patients including multigland disease, persistent or recurrent disease, notwithstanding multiple imaging. 4D-CT scanning has advantages compared with the other modalities in patients including multigland disease, persistent or recurrent disease [13,14]. In our center, 4D-CT scanning has been used for 2 years. In this period, preoperative localization was performed using 4D-CT scanning in seven patients who could not be detected in USG and MIBI. Preoperative localization of the tomography was confirmed through surgery. The sensitivity of this technique was confirmed by the success of the surgery.

Eighty to $85 \%$ of our PHPT patients have parathyroid adenoma followed by primary parathyroid hyperplasia (15\%-20\%) and parathyroid carcinoma (1\%) [1]. In accordance with the literature, pathologic examination revealed single adenoma in $78.8 \%$ of the patients and hyperplasia in $11.7 \%$ and carcinoma in $1 \%$ in our study. The most common location of parathyroid adenomas in our patients was the lower left of the parathyroid, which is in agreement with the literature [15]. In contrast, Afzal et al. [16] reported the right inferior gland as the most common location. In revision surgeries for PHPT, the right superior and inferior gland were the most common sites of pathology.

During the postoperative period, serum calcium and phosphate levels were measured at 1 day, 6 and 12 months after surgery. The measurements revealed that serum calcium and PTH levels showed a slight decrease during the 1-year follow-up period; however, serum phosphate levels did not change significantly during these measurement intervals. Patients should be evaluated individually, preoperatively and postoperatively, because all serum levels measured after parathyroidectomy show different decreasing amounts. These results show that expected serum levels of calcium, phosphate, and PTH may differ for each patient; patients' medical conditions related or unrelated to parathyroid adenoma can vary according to these results.

Among the patients with PHPT, 88 (64.2\%) had no another thyroid pathology, 23 had multinodular goitre, 19 patients had a single nodule, seven had thyroiditis. Five patients underwent total thyroidectomy and one had subtotal thyroidectomy. This shows that the accompanying thyroid pathology should be investigated before parathyroid surgery.

Preoperative imaging modalities is very important in parathyroidectomy surgery. Defining the location of parathyroid pathology before surgery facilitates parathyroidectomy. Routine use of preoperative imaging modalities reduced the risk of complications in our clinic.

\section{CONFLICT OF INTEREST}

No potential conflict of interest relevant to this article was reported.

\section{ORCID}

Berat Demir $\quad$ https://orcid.org/0000-0002-8574-4927

Adem Binnetoglu https://orcid.org/0000-0002-2797-0248

Akın Sahin https://orcid.org/0000-0003-2683-5236

Dilek Gogas Yavuz https://orcid.org/0000-0002-0075-6313

\section{AUTHOR CONTRIBUTIONS}

Conceptualization: BD. Data curation: AS, BD. Formal analysis: BD. Methodology: AB. Project administration: DGY. Visualization: AS. Writing-original draft: BD. Writing-review \& editing: $\mathrm{AB}, \mathrm{DGY}$.

\section{REFERENCES}

1. Binnetoglu A, Binnetoglu A, Gundogdu Y, Baglam T, Sari M. A surgical challange for primary hyperparathyroidism: intravagal parathyroid adenoma. Arch Otolaryngol Rhinol. 2018;4(3):057-060.

2. Bilezikian JP, Khan AA, Potts JT Jr; Third International Workshop on the Management of Asymptomatic Primary Hyperthyroidism. Guidelines for the management of asymptomatic primary hyperparathyroidism: summary statement from the third international workshop. J Clin Endocrinol Metab. 2009 Feb;94(2):335-9.

3. Clark OH. How should patients with primary hyperparathyroidism be treated? J Clin Endocrinol Metab. 2003 Jul;88(7):3011-4.

4. Morris LF, Lee S, Warneke CL, Abadin SS, Suliburk JW, Romero Arenas MA, et al. Fewer adverse events after reoperative parathyroidectomy associated with initial minimally invasive parathyroidectomy. Am J Surg. 2014 Nov;208(5):850-5.

5. Sosa JA, Udelsman R. Minimally invasive parathyroidectomy. Surg Oncol. 2003 Aug;12(2):125-34.

6. Udelsman R, Lin Z, Donovan P. The superiority of minimally invasive parathyroidectomy based on 1650 consecutive patients with primary hyperparathyroidism. Ann Surg. 2011 Mar;253(3):585-91.

7. Coffey RJ, Lee TC, Canary JJ.The surgical treatment of primary hyperparathyroidism: a 20 year experience. Ann Surg. 1977 May;185(5): 518-23.

8. Wilhelm SM,Wang TS, Ruan DT, Lee JA, Asa SL, Duh QY, et al.The American Association of Endocrine Surgeons guidelines for definitive management of primary hyperparathyroidism. JAMA Surg. 2016 Oct;151(10):959-68.

9. Gasparri G. Updates in primary hyperparathyroidism. Updates Surg. 2017 Jun;69(2):217-23.

10. Norman J, Lopez J, Politz D. Abandoning unilateral parathyroidectomy: why we reversed our position after 15,000 parathyroid operations. J Am Coll Surg. 2012 Mar;214(3):260-9.

11. Horanyi J, Duffek L, Szlavik R, Darvas K, Lakatos P, Toth M, et al. Parathyroid surgical failures with misleading falls of intraoperative parathyroid hormone levels. J Endocrinol Invest. 2003 Nov;26(11): 1095-9.

12. Kuzminski SJ, Sosa JA, Hoang JK. Update in parathyroid imaging. Magn Reson Imaging Clin NAm. 2018 Feb;26(1):151-66.

13. Mortenson MM, Evans DB, Lee JE, Hunter GJ, Shellingerhout D, Vu T, et al. Parathyroid exploration in the reoperative neck: improved preoperative localization with 4D-computed tomography. J Am Coll Surg. 2008 May;206(5):888-95.

14. Kelly HR, Hamberg LM, Hunter GJ. 4D-CT for preoperative local- 
ization of abnormal parathyroid glands in patients with hyperparathyroidism: accuracy and ability to stratify patients by unilateral versus bilateral disease in surgery-naive and re-exploration patients. AJNR Am J Neuroradiol. 2014 Jan;35(1):176-81.

15. Bilezikian JP, Silverberg SJ. Clinical practice: asymptomatic primary hyperparathyroidism. N Engl J Med. 2004 Apr;350(17):1746-51. 16. Afzal A, GauharTM, ButtWT, Khawaja AA,Azim KM. Management of hyperparathyroidism: a five year surgical experience. J Pak Med Assoc. 2011 Dec;61(12):1194-8. 